\title{
Cognitive functioning enhancement in older adults: is there an advantage of multicomponent training over Nordic walking?
}

This article was published in the following Dove Press journal:

Clinical Interventions in Aging

Jean-Jacques Temprado ${ }^{1,2}$ Marine Julien-Vintrou ${ }^{1,2}$

Elisa Loddo ${ }^{1,2}$

Jérome Laurin ${ }^{1,2}$

Rita Sleimen-Malkoun ${ }^{1,2}$

'Institut des Sciences du Mouvement, Faculté des Sciences du Sport, AixMarseille Université, Marseille, France;

${ }^{2}$ Centre National de la Recherche

Scientifique, Paris, France
Correspondence: Jean-Jacques Temprado Aix-Marseille Université, Institut des Sciences du Mouvement, Faculté des Sciences du Sport, 163 Avenue de Luminy, BP 910, Marseille 13288, France

Tel +3349 II7 2272

Email jean-jacques.temprado@univ-amu.fr
Introduction: We compared Nordic walking training (NW) to a multicomponent training (MCT) program of an equivalent intensity, in older adults. Our main hypothesis was that MCT would result in larger effects on cognitive processes than NW.

Methods: Thirty-nine healthy older adults, divided into two groups (NW and MCT), took part in the study ( 17 males, 22 females, mean age $=70.8 \pm 0.8$ years). They were tested for cardiovascular fitness, motor fitness and cognitive performance during the two weeks preceding and following the 12-week training session (3 times/week), respectively. For both the NW and MCT interventions, the training sessions were supervised by a trainer. Heart rate of participants was monitored during the sessions and then used to make training loads as similar as possible between the two groups (TRaining IMPulse method).

Results: Results showed that training resulted in better performance for cardiovascular and motor fitness tests. Among these tests, only two revealed a significant difference between the two groups. The NW group progressed more than the MCT group in the 30 Seconds Chair Stand test, while in the One Leg Stance test, the MCT group progressed more. For the cognitive assessment, a significant effect of training was found for executive functions, spatial memory score, and information processing speed response time, with no differences between the two groups.

Conclusion: The study confirmed that physical exercise has a positive impact on cognitive processes with no advantage of MCT intervention over NW training. A possible reason is that NW intervention not only improved cardiovascular capacities, but also motor fitness, including coordination capacities.

Keywords: aerobic training, cognition, motor fitness, Nordic walking, multicomponent training, aging

\section{Introduction}

Numerous studies have shown that engaging older adults in moderate to vigorous aerobic exercise not only benefits cardio-respiratory fitness ${ }^{1,2}$ but also improves cognitive functioning and brain plasticity. ${ }^{3-5}$ Accordingly, the main relevant quantitative determinants of the effects of aerobic exercise - exercise intensity and duration, training frequency, and type of training (treadmill, bicycle, swimming) - were identified, ${ }^{4}$ as well as the mediating mechanisms, including increase in cardiovascular capacities, the production of neurotrophic factors, changes in neural structures, and brain vascularization. ${ }^{5,6}$

In a pioneering study, Voelcker-Rehage et al, ${ }^{7}$ revealed a strong association between motor fitness and cognitive functioning. These results triggered a shift in the 
researchers' focus from quantitative to qualitative determinants of the effects of exercise on cognitive functioning. ${ }^{7,8}$ Indeed, according to current classification (eg, Pesce, 2012; Voelcker-Rehage, 2010) quantitative determinants refer to intensity and duration of effort, frequency, and length of training sessions and programs. Conversely, qualitative determinant refers to the type of movement used during practice that is, simple or guided movements involving automatic control (eg, pedaling) vs complex motor skills and coordinated movements that involve high-level cognitive (executive) functions. ${ }^{8}$

Accordingly, several studies compared the respective effects of aerobic exercise and complex motor skills training on cognitive processes in older adults ${ }^{9}$ (see Levin et al, 2017; Netz, 2019 for reviews ${ }^{10,11}$ ). The underlying assumption was that the former improves cardiovascular fitness, while the latter prominently improves coordination and motor fitness. Results showed that both cardiovascular and coordination training enhanced cognitive functioning, although the mechanisms that underlie performance changes differed depending on the type of intervention. It is noticeable that, in these studies, the intensity of aerobic effort incurred by coordination training was lower than those of the walking intervention, and even too low to improve cardio-respiratory fitness, as demonstrated in other studies. ${ }^{12,13}$ However, in spite of a difference in exercise intensity in the cardiovascular domain, Berryman et al, ${ }^{13}$ reported that, in healthy older adults, complex motor skills training improved executive functions to the same extent as the combination of highintensity aerobic exercise and strength training. Thus, the question remains of whether the effects induced by aerobic exercise and by a circuit training of complex motor skills on cognitive performance could be cumulated.

To address this issue, we compared the effects of Nordic walking training (NW) and those of a multicomponent training (MCT) program on cardiovascular capacities, motor fitness, and cognitive functioning. NW was chosen because, relative to classic walking, it induces higher heart rate (HR), higher oxygen consumption, ${ }^{14}$ and finally results in larger beneficial effects on cardio-respiratory fitness and muscular strength. ${ }^{15,16}$ MCT consisted of practicing complex motor skills requiring coordination, reactivity, agility, balance, and muscle strengthening. Moreover, it was designed so that the repetition of the exercises required an aerobic effort of a comparable intensity to that required by the NW intervention.

We expected that NW and MCT would result in equivalent improvement of cardiovascular fitness, while MCT would lead to larger improvement of motor fitness than
NW. Accordingly, our main hypothesis was that the MCT intervention, combining aerobic exercise and complex motor skill training, would result in larger effects on cognitive functioning than the NW intervention, supposedly implicating aerobic effort alone. To test this hypothesis, we investigated four domains of cognitive functioning (executive functions, spatial memory, visuospatial capacities, information processing speed), thanks to gamified tasks chosen among those of a computerized battery previously validated for cognitive stimulation and rehabilitation (see Materials and methods section).

\section{Materials and methods}

\section{Participants}

Participants were recruited through advertisements in a local newspaper. A total of 60 people volunteered to participate in the study, but after a pre-inclusion meeting with the staff and a medical screening, 41 healthy adults finally took part to the training programs but, due to adverse events during the course of intervention, only 39 were analyzed (17 males, 22 females, mean $=70.8 \pm 0.8$ years).

All subjects provided a written informed consent to: 1) the procedures of the laboratory testing and 2) the training interventions carried out in a sport club by specialized trainers. The protocol was approved by the local ethic committee of Aix-Marseille University and was in accordance with the ethical standards laid down in the Declaration of Helsinki. The main inclusion criteria for the participants were to be aged between 65 and 85 years, and to be sedentary or moderately active. The exclusion criteria were: 1) suffering from metabolic, cardiac or cardiovascular disease, neurological disorder, or any other motor or cognitive restrictions, 2) cognitive impairments, 3) medical contra-indications to planned exercise, or 4) impossibility to commit to a 12 -week exercise program.

In order to assess the level of physical activity of participants, two questionnaires were administered: i) the Ricci and Gagnon sedentary behavior questionnaire (RG; Montreal University, modified by Laureyns and Séné), and ii) the International Physical Activity Questionnaire (IPAQ, French version, July 2003). ${ }^{17}$ According to the obtained scores participants were divided into two groups of equivalent physical activity levels (see Table 1). The cut-off value used to classify participants as sedentary or moderately active was those proposed by Ricci and Gagnon (sedentary $<18$; moderately active $>18$ and $<35$ ). The Montreal Cognitive Assessment (MOCA) was administered in order to evaluate the global cognitive status of the 
Table I Mean and standard deviation of descriptive characteristics of the sample at baseline

\begin{tabular}{|l|l|l|l|}
\hline Variables & MCT (n=19) & NW (n=20) & $P$-value \\
\hline Gender, female $(\mathrm{n})$ & 10 & 12 & - \\
Age (years) & $72.4 \pm 6.3$ & $69.8 \pm 5.9$ & 0.158 \\
BMI $\left(\mathrm{kg} / \mathrm{m}^{2}\right)$ & $25.5 \pm 3.4$ & $26.3 \pm 5$ & 0.715 \\
MOCA & $26.6 \pm 2.5$ & $25.8 \pm 3.6$ & $0.817^{\mathrm{a}}$ \\
IPAQ & $1.6 \pm 0.5$ & $1.4 \pm 0.5$ & 0.105 \\
RG & $20.4 \pm 7.5$ & $19.1 \pm 7.5$ & 0.593 \\
\hline
\end{tabular}

Note: No difference between groups at baseline. ${ }^{a}$ Independent samples $T$-test, otherwise Mann-Whitney $U$ test.

Abbreviations: MCT, multicomponent training; NW, Nordic walking training; BMI, body mass index; MOCA, Montreal Cognitive Assessment; IPAQ, International Physical Activity questionnaire; RG, Ricci and Gagnon sedentary behavior questionnaire.

participants and to detect a possible cognitive decline. ${ }^{18,19}$ Participants with a score below 23 were excluded during the medical screening.

Since most participants were sedentary at the very beginning of the study, to eventual cardiovascular contra-indication to exercise, a medical screening was carried out for each participant, including a sub-maximal aerobic exercise test on a cyclo-ergometer. Termination criterion of the incremental cycling test was fixed reaching the $85 \%$ of the estimated maximal HR. The maximum HR was estimated using the widely used formula of 220 minus age of participants in years (220-age). It is a safe procedure for older participants, though it is less accurate than $\mathrm{HR}_{\max }$ measured during a maximal incremental test. Electrocardiogram was recorded during exercise. HR was measured at rest, during the effort, and 3 minutes post exercise. Initial mechanical power was set at 30 $\mathrm{W}$ for $1 \mathrm{~min}$ and then the workload was increased each minute by $15 \mathrm{~W}$. Adaptation of the exercise protocol was reported by medical doctors for participants older than 80 years old and for some subjects reporting previous cardiovascular problems $(\mathrm{eg}, 30 \mathrm{~W} / 10 \mathrm{~W} / 1 \mathrm{~min} ; 10 \mathrm{~W} / 10 \mathrm{~W} / 1$ $\mathrm{min}$ ). After being included, participants were asked to maintain their lifestyle during the training period and until the last evaluation session was completed.

\section{Procedure}

Participants were tested for cardiovascular fitness, motor fitness, and cognitive performance during the two weeks preceding and following the 12-weeks training, respectively. Before both evaluation sessions, each participant completed a self-report to attest that they did not suffer from cognitive or sensorimotor troubles that might bias their performance in the experimental tasks.

\section{Cardiovascular and motor fitness assessment}

Eight different tests were used to assess cardiovascular fitness, muscular strength, and motor fitness. Five of them were part of the Senior Fitness Test protocol, ${ }^{20}$ while the others were used in previous studies. ${ }^{9}$ Since walking speed is an important indicator of both physical and cognitive health among older adults (eg, Chan and Pin $2019^{21}$ ), we used the 6 minute walking test (6MWT). Participants were asked to walk "as far as possible" during 6 minutes along a corridor 2-m wide on a 50-meter-long race track. The distance covered in the 6 minutes was recorded as well as the mean of HR during the 6 minutes (expressed in percentage of HRmax). No running was allowed. Standardized encouragements - "you're doing well, keep it up" - were given to subjects every minute during the test. HR recording showed that the participants were trying to achieve their maximum performance since the average HR values were between $79 \%$ and $84 \%$ HRmax at both the pre-test and the post-test.

Given that several MCT workouts as well as the use of walking poles required grip strength, maximal grip strength was measured by using the Hand-Grip test (JAMAR ${ }^{\circledR}$ hand dynamometer, force measured in Newton). The highest strength of three performed trials ( $5 \mathrm{~s}$ each with $30 \mathrm{~s}$ of rest) was recorded and averaged. Moreover, 30-Seconds Arm Curl test and 30-Seconds Chair Stand test, ${ }^{22}$ were performed to assess the anaerobic power and fatigue tolerance (force and endurance) of upper limb and lower limb, respectively. For the 30-Seconds Chair Stand test, subjects did not use their arms during movements. For the 30-Seconds Arm Curl test, the weight was $2 \mathrm{~kg}$ for women and $3 \mathrm{~kg}$ for men in accordance with the recommendations of the Senior Fitness Test protocol. In addition, we administered the Timed Up and Go test ${ }^{23}$ and the Single-Leg-Stance test to assess mobility and balance. ${ }^{24}$ Finally, the Stick Fall test allowed assessing reactivity, ${ }^{9}$ and the 30-Seconds Four-Square Stepping Test $\left(\right.$ FSST) ${ }^{25}$ allowed measuring whole-body coordination and agility.

\section{Cognitive assessment}

To assess cognitive performance, we used computerized tests from the PRESCO battery, which has been scientifically validated through different studies (Happy neuron Pro ${ }^{\circledR}$, Scientific Brain Training). Four games were chosen to assess: 1) executive functions, 2) spatial memory, 3) visuospatial capacities, and 4) information processing speed, respectively. These tests were presented by a block of five trials on a 17" screen. The order of presentation was randomized across 
participants. Participants completed the tasks by using the mouse of the computer. A preliminary familiarization (5 minutes) with the computer and mouse was provided to the participants before the evaluation session.

Executive Functions were evaluated through the "Basketball test", which is inspired by the Tour de Hanoï and requires mental reasoning. Specifically, during a trial, a target configuration representing numbered balls of different colors in three baskets was presented to participants. Then, starting from a different configuration, the task was to mentally represent the needed moves to reproduce the target configuration. Participants were asked to report the number of the required ball moves. Six different configurations of four balls were presented (six trials). Time to respond was unlimited. Response time and error rate were recorded for each participant.

\section{Spatial memory}

The test consisted of observing during $30 \mathrm{~s}$ a $5^{*} 5$ cases grid on which were placed two geometric shapes (a circle or a square) of three different colors (black, red, or green). Then, after memorizing the location and colors, the task consisted of reproducing the initial configuration on an empty grid by picking-up with the mouse the appropriate shapes and colors. Two trials were performed. Time to respond was unlimited. Response time and error rate were recorded for each participant.

\section{Visuospatial capacities}

In half of the trials, a photographic view of a scene with different geometric shapes (cubes, cylinders, ...) was presented to participants. Then, a screenshot was displayed from 1 over 8 possible perspectives and the participants had to choose the point of observation corresponding to the displayed view. In the Second half of trials, a given point of observation was indicated and the participants needed to choose the corresponding screenshot amongst eight represented possibilities. Eight different pictures $(2 \times 4)$ were used ( 8 trials). Response time was unlimited. Response time and error rate were recorded for each participant.

\section{Information processing speed}

Participants had to memorize a target fish, then to identify it as fast as possible when it traversed the screen amongst other different fish types. Fifteen different fish types were used (15 trials). Response time and error rate were recorded for each participant.
During a familiarization phase, easy configurations of the different tests were proposed to the participants, in order to ensure that the task was properly understood. During the experiment, the order of presentation of the different tests to the participants was randomized. Participants performed the experimental session without the help of the experimenter and results were recorded for the statistical analysis.

\section{Training interventions}

Participants were semi-randomly divided into groups, which practiced Nordic walking $(\mathrm{NW}, \mathrm{n}=20)$ and Multicomponent Circuit Training (MCT, $n=19$ ) during 12 weeks, three sessions of 60 minutes a week, for a total of 36 sessions (see Figure 1, flow diagram below). The sessions were carried out on monday, thursday, and friday afternoon. One session was deleted for the NW group due to severe raining. We divided the participants into groups on the basis of several criteria, with the aim of creating similar groups (no statistical differences between the groups for all the variables collected during the first interview). The criteria used were: gender, age, MOCA test score, and distance traveled during the 6MWT.

Two specialized trainers, one in NW and the other in MCT were recruited, and the training protocols were defined during several meetings between the scientific staff and the trainers. The NW and MCT groups were divided into two subgroups that practiced the same day with the same trainer. MCT sessions were held in a gymnasium, while the NW sessions were held in a natural park. To ensure compliance with the exercise protocol by the trainers, all the sessions were supervised by research assistants graduate in sport sciences. Some sessions were filmed with the consent of participants in order to be analyzed by the staff. Weekly meetings between the science team and the trainers allowed the exercise protocol to be adjusted, if necessary. For each training protocol, the sessions comprised three phases: i) warm-up (15-20 minutes), ii) working phase (30 minutes), and iii) cooldown (10 minutes). All the participants were provided with a HR monitor (Polar Electro, Kempele, Finland), which was turned on just after the warm-up phase and switched off before the cool down. Data were used to adjust the training loads and make them as similar as possible between the two groups.

\section{Training load}

During each session, exercise intensity was assessed by recording $\mathrm{HR}$ and subjective ratings of perceived exertion (RPE) (Borg CR10 scale). ${ }^{26}$ Participant learned to monitor their HR max, mean HR, and at the end of each session, they 


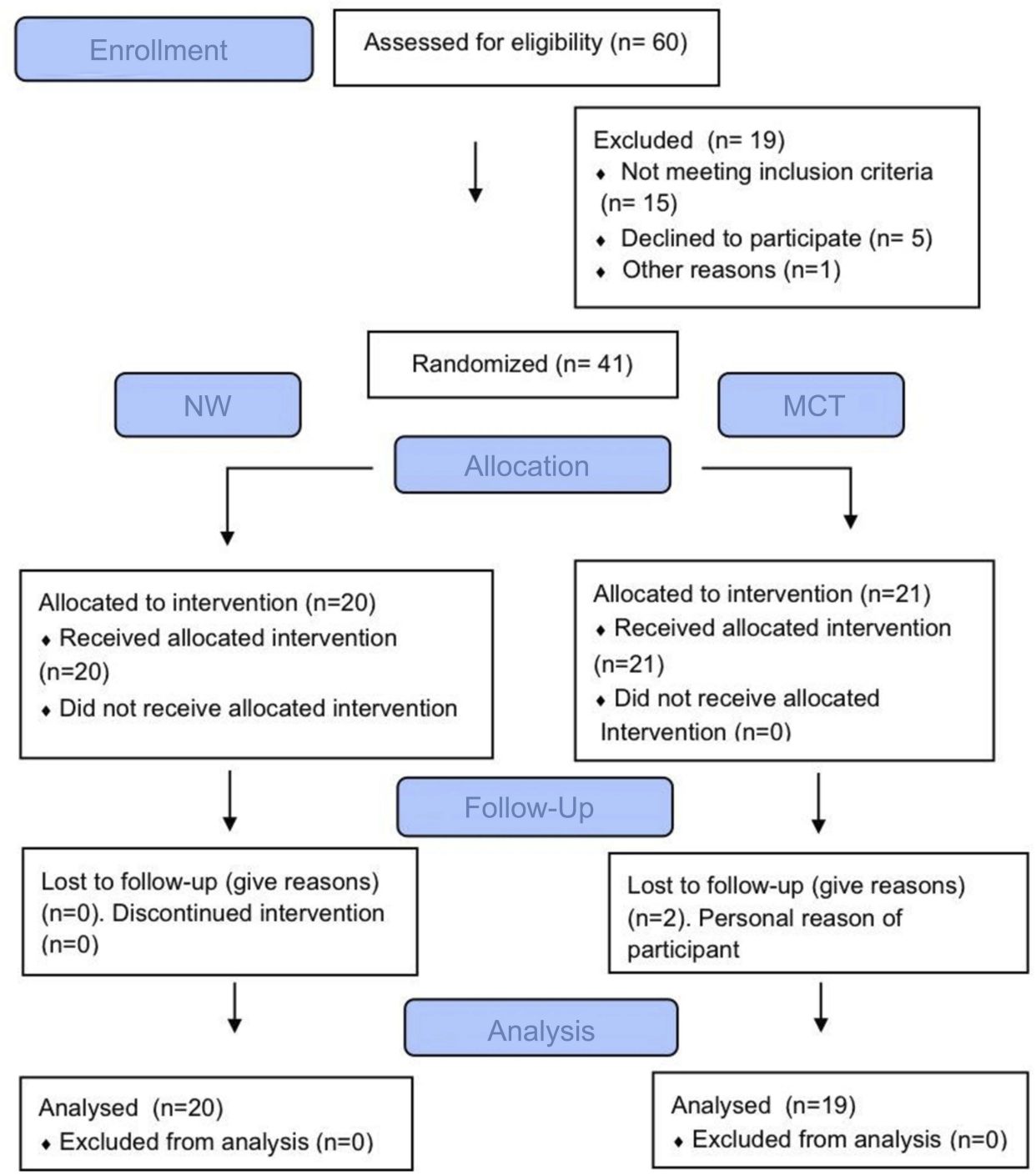

Figure I Study inclusion and exclusion flowchart.

Abbreviations: MCT, multicomponent training; NW, Nordic walking training.

communicate all the information (together with the RPE) to the instructors who registered the data in the training notebook. Participants were instructed to exercise at a moderateintensity, equivalent to a 5-6 on the CR10 scale, and between $60 \%$ and $80 \%$ of the predicted maximal HR.

To compare the training load of MCT and the NW protocols, we use the "training impulse" method (TRIMP). ${ }^{27,28}$ Based on HR, TRIMP method allows approximating the intensity of the session by multiplying the length of the session (in minutes) by an intensity factor which is specifically defined for men and women (Banister's TRIMP $=\mathrm{D} *$ (factor A $* \Delta \mathrm{HR} * \exp$ (factor $\mathrm{B} * \Delta \mathrm{HR})$ ). $\mathrm{D}$ is the duration of training session in minutes, Factor $\mathrm{A}=0.64$ and Factor $\mathrm{B}=1.92$ (for men) and Factor $\mathrm{A}=0.86$ and Factor $\mathrm{B}=1.67$ (for women); $\Delta \mathrm{HR}$ ratio $=($ HRex - HRrest $) /($ HRmax - HRrest $)$, HRex is the average HR recorded during the working period, HR rest is resting HR, and HR max is maximal HR.

\section{Multicomponent circuit training}

The MCT protocol consisted of a circuit of specific exercises. For each exercise, the duration and the number of repetitions were communicated by the trainer to the participants, who could move along the circuit together but still working at their own rate and possibilities. The trainer provided instructions and feedback to participants about the quality of performed movements. Along the 3 months, after every 12 sessions, the exercises were set with different work-to-rest ratio (eg, 1:1, 2:1, or 3:1 minutes), therefore covering both aerobic and anaerobic energy systems. 
Moreover, exercises progressions and variations were modulated over the sessions so as to keep alive the motivation to attend the training program, and continuously challenging participants to practice new motor skills.

The warm-up phase lasted by 15-20 minutes. The main part of the session consisted in moving over 10-11 stations in a prescribed order and a fixed work-to-rest ratio, in which participants had to perform exercise of different nature: muscular resistance for arms and legs, dynamic balance, eye-hand coordination, multi-limb coordination, spatial orientation, reactivity, stepping, and whole-body coordination. The aerobic load of the MCT was induced by intermittent efforts and controlled by HR monitoring. Among the exercises aimed to stimulate motor and cognitive functioning, agility ladder drills consisted in combinations of steps that participants had to repeat all along the ladder, requiring movement speed, rhythms changes, between-leg coordination, change of direction, stopping, postural control, memory recognition, visual attention, and reaction. The ladder drills were modified about every 3 weeks, when the participants mastered movement accuracy and speed. Various drills were proposed along with the whole training program in order to combine wellknown and new exercises in each session. Furthermore, among the exercise focusing on motor and cognitive stimulation were included also ball throwing to small targets and backward locomotion. The cooldown phase consisted in stretching, relaxation, and static balance exercises performed with eyes open and closed. At the end of each session, participants have to reach $60-80 \%$ of their HRmax in order to control the session intensity.

\section{Nordic walking training}

NW consisted in walking with poles continuously around a natural park. Guided by the trainer, during the warm-up phase (10 minutes), participants walked at moderate speed and they performed joints mobilization and general exercises with the help of the poles. In the main phase of the session, participants were instructed to perform moderate- to high-intensity walking for 30 minutes. During the first 2 weeks, participants learned the basic techniques of Nordic walking in flat trails. Afterward, the trainer learned them uphill and downhill techniques in order to be able to follow different trails all over the park. After the first weeks of adaptation, the NW group has increased the intensity of the effort until it reaches by $80 \%$ of the $\mathrm{HR}_{\max }$ in the main part of the session.

\section{Statistical analysis}

Data were analyzed SPPS for MAC, version 24.0 (SPSS Inc., Chicago Illinois). The level of statistical significance was set at $P<0.05$ for all computations. All data were checked for normality of distribution. To test the effects of the training interventions, all the measured variables were submitted to a two-way repeated measures analysis of variance with group (NW vs MCT) as between-subjects factor and session (Pre-test vs Post-test) as within-subjects factor. A Greenhouse-Geisser correction was used in case of violation of the sphericity assumption in ANOVAs. A Greenhouse-Geisser correction was used in case of violation of the sphericity assumption in ANOVAs. Effect sizes were calculated using partial eta squared $\left(\eta_{\mathrm{p}}{ }^{2}\right)$. In addition, for each group, we counted 1) the number of participants who were progressing after training in the different tasks, and 2) the amplitude of progress (in \% of initial performance). Then, we calculated the \% of responders in each group (ie, participants improving their performance by $5 \%$ or more; but see Pickering \& Kielly, $2018^{29}$ for a different method) and the mean amplitude of progress of performance in the progressing participants. Accordingly, the delta value $(\Delta \%)$ between pre-test and post-test was calculated for amplitude of progress $(\Delta \%=$ [post-test score-pretest score)/pre-test score] $* 100)$. Then, the values of observed in NW and MCT groups were compared with an independent samplet-test.

\section{Results}

\section{Attendance to the training programs}

Thirty-six sessions were planned ( 3 sessions $\times 12$ weeks) but only 35 were performed for the NW group and 34 for the MCT group. The attendance to the training program was at least $70 \%$ for all NW group participants, and among them, eight attended more than $90 \%$ of the training sessions. For the MCT group, participation was over $80 \%$ (probably because the sessions took place indoor), and 12 participants achieved more than $90 \%$ attendance. The distribution of attendance to training sessions in each group is shown in Figure 2.

\section{Training load}

The independent samples $t$-test showed a significant difference in the mean training load between the NW and the MCT groups. Specifically, the workload of the NW group was significantly higher than those of the MCT group (39.8 and 25.6, respectively) $(\mathrm{t}(37)=3.98 ; P<0.05)$. This 


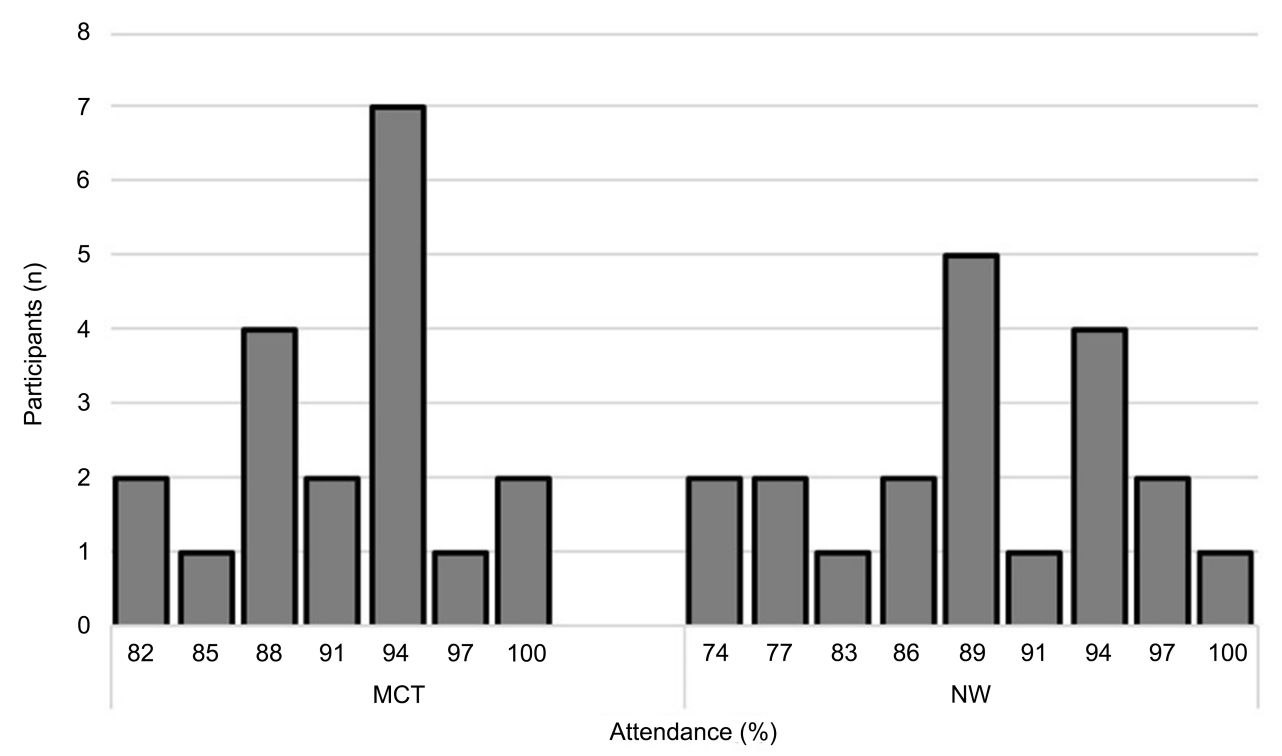

Figure 2 Distribution of attendance to the training sessions in multicomponent training (MCT) group and Nordic walking training (NW) group.

might be induced by a slightly different duration of the sessions between groups. Indeed, the $t$-test showed that the duration of the main phase of the training was significantly longer for the NW group ( $\mathrm{M}=29.1$ minutes) than for the MCT group ( $\mathrm{M}=25.3$ minutes) $(\mathrm{t}(136)=3.49 ; P<0.05)$.

\section{Cardiovascular and motor fitness}

Repeated measure ANOVA revealed a main effect of session for all the different tests, that is: the 30 Seconds Chair Stand $\left[\mathrm{F}(1.37)=5.342, P<0.05, \eta_{\mathrm{p}}{ }^{2}=0.126\right]$, the One Leg Stance $\left[\mathrm{F}(1.37)=10.589, \quad P<0.05, \quad \eta_{\mathrm{p}}{ }^{2}=0.223\right]$, the 30 Seconds Arm Curl [F(1.37)=19.742, $\left.P<0.05, \eta_{\mathrm{p}}{ }^{2}=0.348\right]$, Timed Up and Go $\left[\mathrm{F}(1.37)=8.141, P<0.05, \eta_{\mathrm{p}}{ }^{2}=0.180\right]$, 6MWT $\left[\mathrm{F}(1.37)=21.059, P<0.05, \eta_{\mathrm{p}}{ }^{2}=0.363\right]$, Hand Grip Strength $\left[\left(\mathrm{F}(1.37)=18.812, P<0.05, \eta_{\mathrm{p}}{ }^{2}=0.337\right]\right.$, Stick Fall test $\left[\left(\mathrm{F}(1.37)=8.041, P<0.05, \eta_{\mathrm{p}}{ }^{2}=0.179\right]\right.$, and for the 30 Seconds FSST $\left[\left(\mathrm{F}(1.37)=33.702, P<0.05, \eta_{\mathrm{p}}{ }^{2}=0.477\right]\right.$. These results showed increased performance after the training sessions for all the different tests. Among these tests, only two revealed a significant session $\dot{x}$ group interaction that is: the 30 Seconds Chair Stand $[(\mathrm{F}(1.37)$ $\left.=6.669, P<0.05, \eta_{\mathrm{p}}{ }^{2}=0.153\right]$ and the One Leg Stance $[\mathrm{F}$ $\left.\left.(1.37)=8.678, P<0.05, \eta_{\mathrm{p}}{ }^{2}=0.190\right)\right]$, respectively. The independent sample $t$-test showed that in the 30 Seconds Chair Stand, the NW group $(\mathrm{M}=19.2, \mathrm{SD}=26.5)$ progressed more than the MCT group $(\mathrm{M}=3.9, \mathrm{SD}=16.6) ; \mathrm{t}(37)=-2.147$, $P<0.05$, while for the One Leg Stance, the MCT group $(\mathrm{M}=237.3, \mathrm{SD}=254.8)$ progressed more than the NW group $\quad(\mathrm{M}=64.2, \quad \mathrm{SD}=167.8), \quad \mathrm{t}(37)=2.324, \quad P<0.05)$.
Figure 3 shows the mean pre- to post-intervention changes on motor outcomes (ie $\Delta$ performance pre-post).

In addition, we counted the number of participants who progressed ("responders to exercise") in each group. Although on average, this number was high and nearly similar between the NW and the MCT groups $(73.1 \%$ and $70.6 \%$, respectively), group differences were observed as a function of the nature of the test. For instance, in the 6MWT, the percentage of responders was higher in the NW group than in the MCT group ( $90 \%$ and $79 \%$, respectively), while the inverse was observed for the one-leg stance test $(60 \%$ and $81 \%$, respectively). For the other tests, the percentage of responders was roughly similar between the two groups. The analysis conducted on the mean amplitude of progress on cardiovascular and motor performance within the progressing participants revealed a significant difference only in One Leg Stance performance, where the progress of the MCT $(\mathrm{M}=352.6, \mathrm{SD}=227.3)$ was significantly larger than the one of the NW (M=153.3, $\mathrm{SD}=202.8), \mathrm{t}(19)=-2.029, P<0.05$. Table 2 summarizes the number of participants that improved their performance in the fitness tests and the corresponding amplitude of progress.

\section{Cognitive performance}

Significant session effect on error rate was observed for executive functions $\left[\mathrm{F}(1.36)=4.24, \quad P<0.04, \eta^{2}=0.106\right]$, and spatial memory $\left[\mathrm{F}(1.36)=4.049, P<0.05, \eta_{\mathrm{p}}{ }^{2}=0.101\right]$. No session $\dot{x}$ group interaction was found for these tests. For the information processing speed test, the analysis 


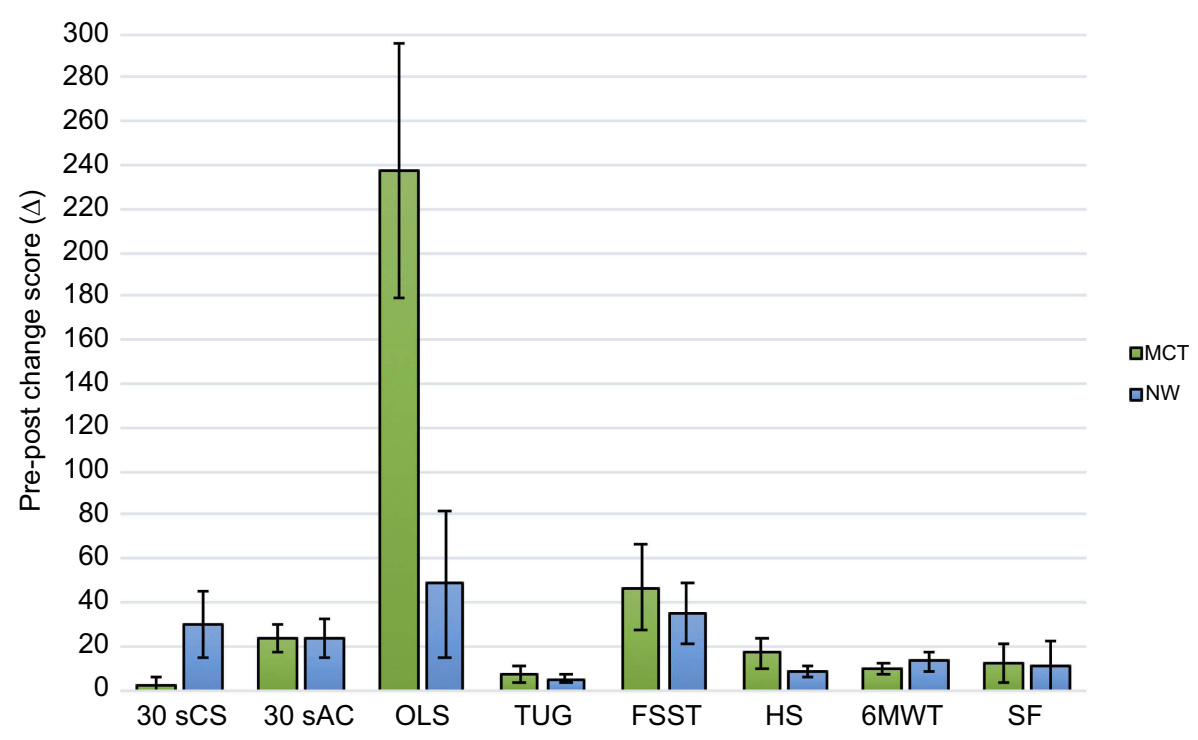

Figure 3 Mean pre- to post-intervention change scores $(\Delta)$ on motor outcomes for participants completing the multicomponent training (MCT) and the Nordic walking training (NW).

Abbreviations: 30sCS, 30 Seconds Chair Stand Tests; 30sAC, 30 Seconds Arm Curl Tests; OLS, one leg stance test; TUG, timed up and go test; FSST, four square step tests; HS, hand grip strength test; 6MWT, 6 minute walking test; SF, stick fall test.

revealed a significant effect of session on response time $[\mathrm{F}$ $\left.(1.36)=6.993, P<0.05, \eta_{\mathrm{p}}{ }^{2}=0.159\right]$.

The counting of the number of responders in each group (ie, $\Delta$ performance pre-post $>1 \%$ ) revelated that, for the executive functions test, the percentage of responders was higher for the MCT group (55\%) than for the NW group (30\%). For the other tests, the percentage of responders was roughly similar between the two groups (see Table 3). For the mean amplitude of progress in the test of executive functions, the independent $t$-test showed that the progress of the NW $(\mathrm{M}=58.1, \mathrm{SD}=22.9)$ was larger compared to the MCT $(\mathrm{M}=31, \mathrm{SD}=11.5), \mathrm{t}(6.55)=2.696$, $P<0.05$. Levene's test indicated unequal variances $(\mathrm{F}=7.561, p=0.016)$, so degrees of freedom were adjusted from 14 to 6.551 . For the other tests, the independent $t$-test has not revealed significant differences between MCT and NW. Table 3 summarizes the number of participants that improved their performance in the cognitive tests and the corresponding mean amplitude of progress (Table 4).

\section{Discussion}

We aimed to compare a NW intervention with a multicomponent training program in order to determine whether combining aerobic exercise and complex motor skills training (MCT) had an advantage over aerobic exercise alone (NW) to enhance cognitive functioning. Our main hypothesis was that MCT would result in larger effects on cognitive processes than NW. Also, we predicted that NW and MCT would result in equivalent improvement of cardiovascular fitness, while MCT would lead to larger improvement of motor fitness than NW.

\section{Effects of training programs on cardiovascular and motor fitness}

Results observed in the 6MWT confirmed that the participants of both groups significantly improved their performance without any difference between them at the end of the training period. Thus, although slightly differently stimulated during training (as attested that the results of training load), the NW and MCT interventions led to comparable progress of performance. These results were pre-requisite to further compare the effects of aerobic training and complex motor skill training on cognitive processes. In this respect, the present study differs from those available in the literature, which have shown that multicomponent training was too low to improve cardiorespiratory fitness. ${ }^{12,13,30}$ However, the number of responders was larger in the NW groups than in the MCT group ( $90 \%$ and $79 \%$, respectively), thereby suggesting that NW might be slightly more effective than MCT to enhance cardiovascular fitness.

Concerning motor fitness, the results showed that the two groups progressed in all the different tests. In addition, the differences in performance observed in the 30 Seconds Chair Stand test and the One Leg Stance test revealed the specificity of each training intervention. Specifically, the 


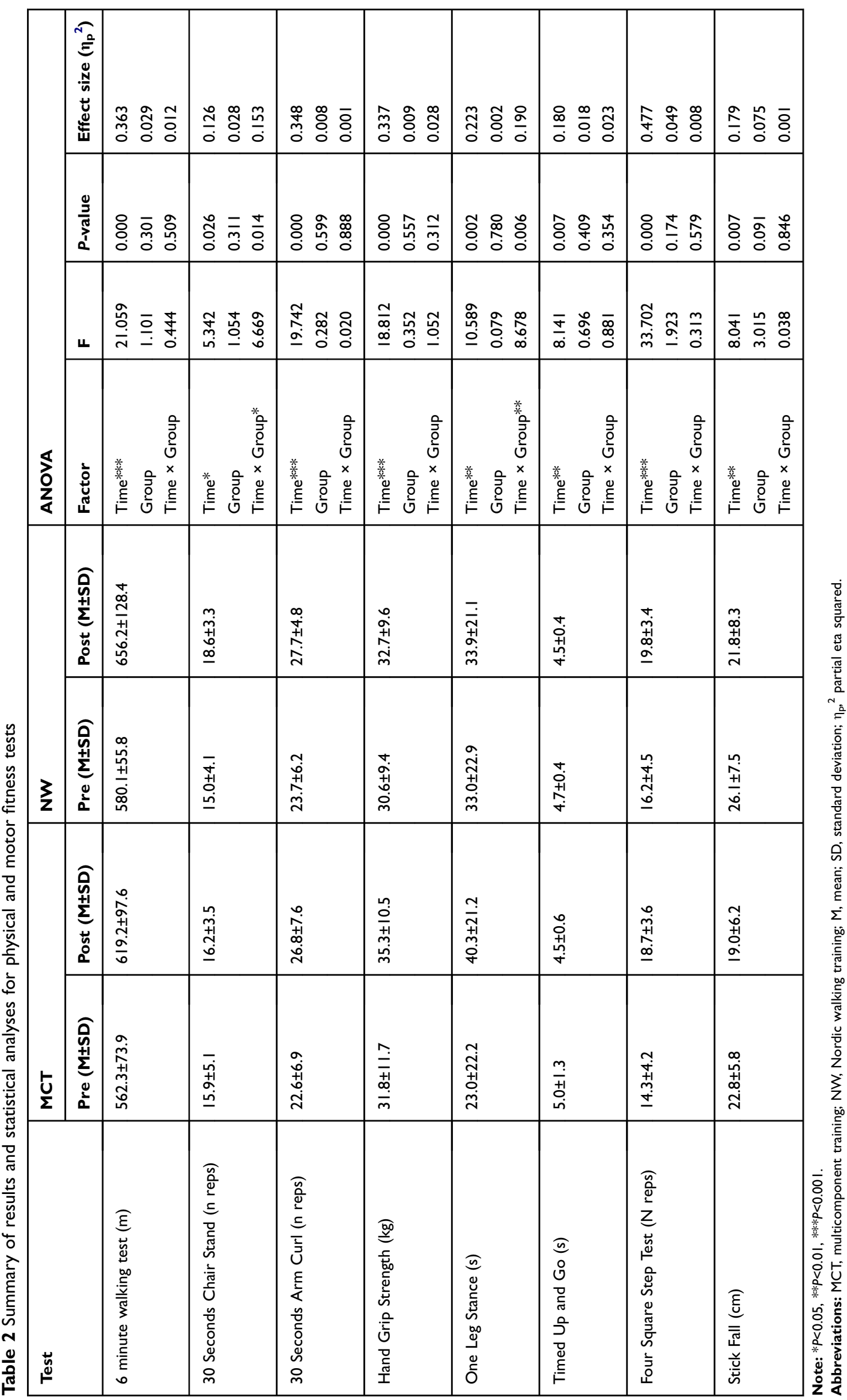


Table 3 Mean pre- to post-intervention change score $(\Delta)$ on motor outcomes for participants who progressed following 12 weeks of multicomponent training (MCT) and Nordic walking training (NW)

\begin{tabular}{|l|l|l|l|l|}
\hline \multirow{2}{*}{ Test } & \multicolumn{2}{l|}{ MCT } & \multicolumn{2}{l|}{ NW } \\
\cline { 2 - 5 } & $\begin{array}{l}\text { Progr- } \\
\text { essors } \\
\text { n (\%) }\end{array}$ & $\begin{array}{l}\Delta \\
\text { (\%) }\end{array}$ & $\begin{array}{l}\text { Progr- } \\
\text { essors } \\
\text { n (\%) }\end{array}$ & $\begin{array}{l}\Delta \\
\text { (\%) }\end{array}$ \\
\hline 6 minute walking test (m) & 79 & 13 & 90 & 17 \\
30 Seconds Chair Stand (n reps) & 32 & 26 & 80 & 28 \\
30 Seconds Arm Curl (n reps) & 74 & 35 & 70 & 28 \\
Hand Grip Strength (Kg) & 68 & 20 & 75 & 14 \\
One Leg Stance (s) & 81 & 220 & 60 & 58 \\
Timed Up and Go (s) & 68 & 17 & 60 & 12 \\
Four Square Step Test (N reps) & 84 & 37 & 80 & 39 \\
Stick Fall (cm) & 79 & 27 & 70 & 31 \\
\hline
\end{tabular}

Abbreviation: $\mathrm{n}$ reps, number of repetitions.

Table 4 Mean pre- to post-intervention change score $(\Delta)$ on cognitive outcomes for participants who progressed after the multicomponent training (MCT) and the Nordic walking training (NW)

\begin{tabular}{|l|l|l|l|l|}
\hline \multirow{2}{*}{ Outcomes } & \multicolumn{2}{|l|}{ MCT } & \multicolumn{2}{l|}{ NW } \\
\cline { 2 - 5 } & $\begin{array}{l}\text { Progressors } \\
\text { n (\%) }\end{array}$ & $\begin{array}{l}\Delta \\
\text { (\%) }\end{array}$ & $\begin{array}{l}\text { Progressors } \\
\text { n (\%) }\end{array}$ & $\begin{array}{l}\Delta \\
\text { (\%) }\end{array}$ \\
\hline Executive Functions & 56 & 31 & 30 & 58.2 \\
Spatial Memory & 61 & 26.6 & 65 & 36.4 \\
Visuospatial & 39 & 20.9 & 40 & 27.9 \\
Capacities & & & & \\
Information & 37 & 19 & 45 & 17.6 \\
Processing Speed & & & & \\
\hline
\end{tabular}

NW group progressed more in the 30 Seconds Chair Stand test, which was related to force capacities of lower limbs, while the MCT group progressed more in the One Leg Stance test, which assessed dynamic balance considered as a component of motor fitness. The lack of difference in the 30-Seconds FSST suggests that NW also improved motor fitness and, in particular, motor coordination. This result was unexpected since the participants of the MCT training group spent a lot of time on practicing coordination tasks over the different sessions, in particular through agility ladder drills. In addition, previous studies have shown that NW prominently improves cardiovascular fitness instead of motor fitness. ${ }^{16,31}$ A possible interpretation is that the two training programs allowed developing coordination skills through different pathways. Presumably, the
NW program was prominently based on the repetition of arm-leg coordination over a large number of repetitions (quantitative), while the MCT program allowed developing whole-body and multi-limb coordination through a variety of qualitative exercises. Thus, it seems that NW and MCT are different training programs, both qualitatively and quantitatively, which equally can develop motor fitness and coordination capacities.

\section{Effects of NW and MCT on cognition}

Both the MCT and the NW participants progressed in executive functions, spatial memory, and information processing speed tests as a consequence of physical training. On average, the performance in these three tests did not differ between the two groups. Nevertheless, when considering only the responders, the results showed a differentiation between the two groups in the test of executive functions. Indeed, on the one hand, the MCT program allowed more participants to progress, while, on the other hand, for the responders, the NW program induced a greater improvement in performance in the test of executive functions. This result might suggest that the two intervention programs might be slightly different in the way they impacted executive functions. Presumably, on the one hand, the benefits of NW on executive functions resulted from indirect effects of aerobic effort on cognition-related brain structure. On the one hand, in addition to the indirect effects of aerobic exercise on cognition-related brain structures, MCT prominently directly loaded executive functions through the practice complex movements. Such hypothesis was also supported by the results observed in Voelcker-Rehage et al's study, ${ }^{9}$ which showed that aerobic intervention and coordination training have different effects on cognitive performance. Accordingly, in our study, the differences observed for the sub-groups of responders on cognitive performance could reflect different underlying mechanisms involved, respectively, in NW and MCT. However, it cannot be excluded that NW also directly loaded cognitive processes, which are critical to successfully achieve walking. In a systematic review, Hamacher et $\mathrm{al}^{32}{ }^{32}$ provided supporting evidence of this hypothesis that, in older adults, executive functions are used to achieve normal locomotion function, in particular when they walk fast. Finally, the present study adds to the evidence that physical exercise has a positive impact on cognitive processes (see also Hamacher et $\mathrm{al}^{32}{ }^{3}$ ). It also suggests that NW can be considered as a multi-component intervention, which may have greater effect than aerobic 
exercise alone. Since in the present experiment, no comparison was made with a control group following aerobic exercise alone, this conclusion remains speculative. However, consistent results have been reported in this respect by Hamacher et $\mathrm{al}^{33}$ with dancing intervention, which can also be considered as a multicomponent activity. In this study, greater effects of dancing intervention were found, compared to aerobic training.

\section{Conclusion}

Contrary to our initial hypothesis, MCT intervention did not enhance cognitive processes better than the NW training. On the basis of this result, it could be concluded that the effects of aerobic training and complex motor skill training do not cumulate to improve cognition. However, this conclusion is equivocal since the NW intervention improved both cardiovascular capacities and motor fitness, including coordination. This result differs from those reported in previous studies, which showed beneficial effects of NW on cardio-respiratory fitness and muscular strength instead of motor fitness. ${ }^{16,34}$ In the present study, motor coordination involved in walking with poles and, more generally, the improvement of motor fitness induced by NW may have also contributed to reduce the difference between the effects of the two intervention programs on cognitive functioning. This could be attributed to the fact that all the sessions of NW were carried out in natural environment and complex terrains (uneven surfaces, gravel, rocks ... ), which probably contributed to increase balance, motor, and even cognitive demands.

Thus, whether the effects of a pure aerobic exercise and the practice of complex motor skills on cognitive processes can be cumulated remains unknown and deserves to be further investigated. Another limitation of the present study is that it has no control group. Thus, it is possible that some of the progress observed for the two groups results from the test-retest effect.

However, this study adds a significant contribution to the existing literature that has important practical consequences. Indeed, NW is a form of exercise that has increasingly gained interest among middle-aged and elderly people during the last few years. A frequently evoked reason is that NW poles are a useful equipment for supporting walking exercise, which reinforce balance control and leads to significant cardiovascular benefits by increasing oxygen use and caloric expenditure compared to regular walking. ${ }^{35-37}$ The present study also demonstrated that Nordic walking improves motor fitness and coordination. Thus, our results lead to conclude that Nordic walking can be highly recommended as an effective and efficient mode of exercise in older adults to improve cardiovascular capacities, motor fitness, including coordination capacities, and last but not least, to enhance cognitive functioning.

\section{Acknowledgments}

The present study was financially supported by AG2R-LA MONDIALE. Medical screening was done by doctors of the Sport Medicine Department of Assistance Publique Hôpitaux de Marseille (JM Coudreuse and D Marziale) and Hôpital Européen (G Lagrange). We thank Jean-Pierre Camm, Matthieu Gonet, Isabelle Petit and the Stade Marseillais Universite Club for the implementation and supervision of physical activity programs. We express our gratitude to the participants.

\section{Author contributions}

JJ Temprado and E Loddo wrote the first draft of the paper. All the authors contributed to data recording, data analysis, and critically revising the paper, gave final approval of the version to be published, and agreed to be accountable for all aspects of the work.

\section{Disclosure}

The authors report no conflict of interests in this work.

\section{References}

1. Kasch FW, Boyer JL, Schmidt PK, et al. Ageing of the cardiovascular system during 33 years of aerobic exercise. Age Ageing. 1999;28 (6):531-536. doi:10.1093/ageing/28.6.531

2. Takeshima N, Rogers ME, Islam MM, Yamauchi T, Watanabe E, Okada A. Effect of concurrent aerobic and resistance circuit exercise training on fitness in older adults. Eur J Appl Physiol. 2004;93(12):173-182. doi:10.1007/s00421-004-1193-3

3. Bherer L, Erickson KI, Liu-Ambrose T. A Review of the effects of physical activity and exercise on cognitive and brain functions in older adults. J Aging Res. 2013;2013. doi:10.1155/2013/657508

4. Colcombe S, Kramer AF. Fitness effects on the cognitive function of older adults: a meta-analytic study. Psychol Sci. 2003;14(2):125-130. doi:10.1111/1467-9280.t01-1-01430

5. Hillman CH, Erickson KI, Kramer AF. Be smart, exercise your heart: exercise effects on brain and cognition. Nature Rev Neurosci. 2008;9 (1):58-65. doi: $10.1038 / \mathrm{nrn} 2298$

6. Etnier JL, Chang Y-K. The effect of physical activity on executive function: a brief commentary on definitions, measurement issues, and the current state of the literature. J Sport Exerc Psychol. 2009;31 (4):469-483.

7. Voelcker-Rehage C, Godde B, Staudinger UM. Physical and motor fitness are both related to cognition in old age. Eur $J$ Neurosci. 2010;31(1):167-176. doi:10.1111/j.1460-9568.2009.07014.x 
8. Pesce C. Shifting the focus from quantitative to qualitative exercise characteristics in exercise and cognition research. J Sport Exerc Psychol. 2012;34(6):766-786.

9. Voelcker-Rehage C, Godde B, Staudinger UM. Cardiovascular and coordination training differentially improve cognitive performance and neural processing in older adults. Front Hum Neurosci. 2011;5. doi:10.3389/fnhum.2011.00026

10. Levin O, Netz Y, Ziv G. The beneficial effects of different types of exercise interventions on motor and cognitive functions in older age: a systematic review. Eur Rev Aging Phys Act. 2017;14. doi:10.1186/s11556-017-0189-z

11. Netz Y. Is there a preferred mode of exercise for cognition enhancement in older age? - a narrative review. Front Med (Lausanne). 2019;6. doi:10.3389/fmed.2019.00057

12. Arrieta H, Rezola-Pardo C, Echeverria I, et al. Physical activity and fitness are associated with verbal memory, quality of life and depression among nursing home residents: preliminary data of a randomized controlled trial. BMC Geriatr. 2018;18. doi:10.1186/s12877-018-0770-y

13. Berryman N, Bherer L, Nadeau S, et al. Multiple roads lead to Rome: combined high-intensity aerobic and strength training vs. gross motor activities leads to equivalent improvement in executive functions in a cohort of healthy older adults. Age (Dordr). 2014;36(5). doi:10.1007/ s11357-014-9710-8

14. Schiffer T, Knicker A, Hoffman U, Harwig B, Hollmann W, Strüder HK. Physiological responses to nordic walking, walking and jogging. Eur $J$ Appl Physiol. 2006;98(1):56-61. doi:10.1007/s00421-006-0242-5

15. Takeshima N, Rogers NL, Rogers ME, Islam MM, Koizumi D, Lee S. Functional fitness gain varies in older adults depending on exercise mode. Med Sci Sports Exerc. 2007;39(11):2036-2043. doi:10.1249/ mss.0b013e31814844b7

16. Takeshima N, Islam MM, Rogers ME, et al. Effects of nordic walking compared to conventional walking and band-based resistance exercise on fitness in older adults. J Sports Sci Med. 2013;12 (3):422-430.

17. Craig CL, Marshall AL, Sjöström M, et al. International physical activity questionnaire: 12-country reliability and validity. Med Sci Sports Exerc. 2003;35(8):1381-1395. doi:10.1249/01.MSS.0000078924.61453.FB

18. Nasreddine ZS, Phillips NA, Bédirian V, et al. The Montreal Cognitive Assessment, MoCA: a brief screening tool for mild cognitive impairment. $J$ Am Geriatr Soc. 2005;53(4):695-699. doi:10.1111/j.1532-5415.2005.53221.x

19. Nasreddine ZS, Patel BB. Validation of Montreal Cognitive Assessment, MoCA, alternate french versions. Can J Neurol Sci. 2016;43(5):665-671. doi:10.1017/cjn.2016.273

20. Rikli RE, Jones CJ. Development and validation of criterion-referenced clinically relevant fitness standards for maintaining physical independence in later years. Gerontologist. 2013;53(2):255-267. doi:10.1093/geront/gns071

21. Chan WLS, Pin TW. Reliability, validity and minimal detectable change of 2 min walk test, 6 min walk test and 10-meter walk test in frail older adults with dementia. Exp Gerontol. 2019;115:9-18. doi:10.1016/j.exger.2018.11.001
22. Jones CJ, Rikli RE, Beam WC. A 30-s Chair-Stand Test as a measure of lower body strength in community-residing older adults. Res $Q$ Exerc Sport. 1999;70(2):113-119. doi:10.1080/02701367.1999.10608028

23. Podsiadlo D, Richardson S. The timed "Up \& Go": a test of basic functional mobility for frail elderly persons. $J$ Am Geriatr Soc. 1991;39(2):142-148.

24. Springer BA, Marin R, Cyhan T, Roberts H, Gill NW. Normative values for the unipedal stance test with eyes open and closed. $J$ Geriatr Phys Ther. 2007;30(1):8-15.

25. Dite W, Temple VA. A clinical test of stepping and change of direction to identify multiple falling older adults. Arch Phys Med Rehabil. 2002;83(11):1566-1571. doi:10.1053/apmr.2002.35469

26. Borg GA. Psychophysical bases of perceived exertion. Med Sci Sports Exerc. 1982;14(5):377-381.

27. Banister EW, Calvert TW. Planning for future performance: implications for long term training. Can J Appl Sport Sci. 1980;5(3):170-176.

28. Borresen J, Lambert MI. The quantification of training load, the training response and the effect on performance. Sports Med. 2009;39(9):779-795. doi:10.2165/11317780-000000000-00000

29. Pickering C, Kiely J. Do non-responders to exercise exist-and if so, what should we do about them? Sports Med. 2019;49(1):1-7. doi:10.1007/s40279-018-01041-1

30. Godde B, Voelcker-Rehage C. Cognitive resources necessary for motor control in older adults are reduced by walking and coordination training. Front Hum Neurosci. 2017;11:156. doi:10.3389/ fnhum.2017.00156

31. Gomeñuka NA, Oliveira HB, Silva ES, et al. Effects of Nordic walking training on quality of life, balance and functional mobility in elderly: a randomized clinical trial. PLoS One. 2019;14:1. doi:10.1371/journal.pone.0211472

32. Hamacher D, Herold F, Wiegel P, Hamacher D, Schega L. Brain activity during walking: a systematic review. Neurosci Biobehav Rev. 2015;57:310-327. doi:10.1016/j.neubiorev.2015.08.002

33. Hamacher D, Hamacher D, Rehfeld K, Hökelmann A, Schega L. The effect of a six-month dancing program on motor-cognitive dual-task performance in older adults. J Aging Phys Act. 2015;23(4):647-652. doi:10.1123/japa.2014-0067

34. Chodzko-Zajko WJ, Proctor DN, Fiatarone Singh MA, et al. Exercise and physical activity for older adults. Med Sci Sports Exerc. 2009;41 (7):1510. doi:10.1249/MSS.0b013e3181a0c95c

35. Church TS, Earnest CP, Morss GM. Field testing of physiological responses associated with Nordic Walking. Res $Q$ Exerc Sport. 2002;73(3):296-300. doi:10.1080/02701367.2002.10609023

36. Kukkonen-Harjula K, Hiilloskorpi H, Mänttäri A, et al. Self-guided brisk walking training with or without poles: a randomized-controlled trial in middle-aged women. Scand J Med Sci Sports. 2007;17 (4):316-323. doi:10.1111/j.1600-0838.2006.00585.x

37. Porcari JP, Hendrickson TL, Walter PR, Terry L, Walsko G. The physiological responses to walking with and without Power Poles on treadmill exercise. Res $Q$ Exerc Sport. 1997;68(2):161-166. doi:10.1080/02701367.1997.10607992
Clinical Interventions in Aging

\section{Publish your work in this journal}

Clinical Interventions in Aging is an international, peer-reviewed journal focusing on evidence-based reports on the value or lack thereof of treatments intended to prevent or delay the onset of maladaptive correlates of aging in human beings. This journal is indexed on PubMed Central, MedLine, CAS, Scopus and the Elsevier
Bibliographic databases. The manuscript management system is completely online and includes a very quick and fair peer-review system, which is all easy to use. Visit http://www.dovepress.com/ testimonials.php to read real quotes from published authors. 